RIMS-1668

Submodular Function Minimization under Covering Constraints

By

Satoru IWATA and Kiyohito NAGANO

April 2009

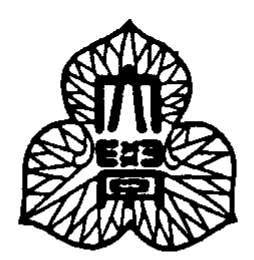

京都大学 数理解析研究所

RESEARCH INSTITUTE FOR MATHEMATICAL SCIENCES

KYOTO UNIVERSITY, Kyoto, Japan 


\title{
Submodular Function Minimization under Covering Constraints
}

\author{
Satoru Iwata* $\quad$ Kiyohito Nagano ${ }^{\dagger}$
}

April 2009

\begin{abstract}
This paper addresses the problems of minimizing nonnegative submodular functions under covering constraints, which generalize the vertex cover, edge cover, and set cover problems. We give approximation algorithms for these problems exploiting the discrete convexity of submodular functions. We first present a rounding 2-approximation algorithm for the submodular vertex cover problem based on the half-integrality of the continuous relaxation problem, and show that the rounding algorithm can be performed by one application of submodular function minimization on a ring family. We also show that a rounding algorithm and a primal-dual algorithm for the submodular cost set cover problem are both constant factor approximation algorithms if the maximum frequency is fixed. In addition, we give an essentially tight lower bound on the approximability of the submodular edge cover problem.
\end{abstract}

\section{Introduction}

Let $N$ be a finite nonempty set of cardinality $n$. A real-valued set function $\rho$ on $N$ is submodular if it satisfies

$$
\rho(X)+\rho(Y) \geq \rho(X \cup Y)+\rho(X \cap Y), \quad X, Y \subseteq N .
$$

In the areas of combinatorial optimization, game theory, and machine learning and various other fields, submodular set functions are recognized as fundamental tools and interesting subjects of research. Besides, submodular functions and convex functions are closely related: a set function is submodular if and only if its Lovász extension is convex [19].

The first polynomial algorithm for submodular function minimization, described by Grötschel, Lovász, and Schrijver [10,11], relies on the ellipsoid method. Combinatorial strongly polynomial algorithms for minimizing submodular functions were developed later by Iwata, Fleischer, and Fujishige [15] and by Schrijver [26]. These combinatorial algorithms have been improved in time complexity $[14,16,25]$.

\footnotetext{
${ }^{*}$ Research Institute for Mathematical Sciences, Kyoto University, Kyoto 606-8502, Japan (E-mail: iwata@kurims.kyoto-u.ac.jp). Research supported by the Asahi Glass Foundation and the Kayamori Foundation.

${ }^{\dagger}$ Department of Mathematical and Computing Sciences, Graduate School of Information Science and Engineering, Tokyo Institute of Technology, Tokyo 152-8552, Japan (E-mail: nagano@is.titech.ac.jp).
} 
In contrast, the maximization problem of submodular functions is NP-hard, as it contains the maximum cut problem. Approximation algorithms for the maximization have been extensively studied even under some constraints including knapsack and matroid constraints [6, 18, 30].

Constrained submodular function minimization problems have also been investigated in various contexts. It is easy to see that we can find a nonempty proper subset $X$ that minimizes $\rho(X)$ in polynomial time. When the feasible region $\mathcal{F} \subseteq 2^{N}$ is a ring family, that is, $\mathcal{F}$ is a collection of subsets of $N$ closed with respect to union and intersection, the minimization problem can be solved in polynomial time (see [26]). Goemans and Ramakrishnan [9] dealt with the case when the feasible region is a parity family. Recently, Svitkina and Fleischer [28] considered the submodular function minimization problem with cardinality lower bound and gave an $o(\sqrt{n / \ln n})$ lower bound for the approximability.

This paper addresses the problems of minimizing nonnegative submodular functions under covering constraints. These problems described below generalize the classical covering problems: the set cover, vertex cover, edge cover problems.

Submodular Cost Set Cover: Let $U$ be a finite set of cardinality $k$ and $\mathcal{S}=\left\{S_{1}, \ldots, S_{n}\right\}$ be a collection of its subsets indexed by $N=\{1, \ldots, n\}$. For a subset $X \subseteq N$ we denote $S_{X}=\bigcup\left\{S_{i} \mid i \in X\right\}$. We say that a subset $X \subseteq N$ is a set cover if $S_{X}=U$. Given a nonnegative cost function $c: N \rightarrow \mathbf{R}_{+}$, the set cover problem asks for finding a set cover $X \subseteq N$ that minimizes the cost $c(X)=\sum_{i \in X} c(i)$. This problem is known to be solved approximately in polynomial time within a factor of $\mathrm{O}(\ln k)$ or the maximum frequency $\eta=\max _{u \in U}\left|\left\{i \mid u \in S_{i}\right\}\right|$. Given a nonnegative submodular function $\rho: 2^{N} \rightarrow \mathbf{R}_{+}$, the submodular set cover problem asks for finding a set cover $X \subseteq N$ that minimizes the cost $\rho(X)$.

Submodular Vertex Cover: Let $G=(V, E)$ be a graph with vertex set $V$ and edge set $E$. A vertex subset $X \subseteq V$ is called a vertex cover in $G$ if every edge in $E$ is incident to a vertex in $X$. Given a nonnegative cost function $c: V \rightarrow \mathbf{R}_{+}$, the vertex cover problem asks for finding a vertex cover $X \subseteq V$ that minimizes the cost $c(X)=\sum_{v \in X} c(v)$. This problem is known to be NP-hard, and efficient 2-approximation algorithms are known [1]. Given a nonnegative submodular function $\rho: 2^{V} \rightarrow \mathbf{R}_{+}$, the submodular vertex cover problem asks for finding a vertex cover $X \subseteq V$ that minimizes the cost $\rho(X)$. This is a special case of a submodular cost set cover problem with $U=E$ and $N=V$.

Submodular Edge Cover: Let $H=(W, F)$ be a graph with vertex set $W$ and edge set $F$. An edge subset $X \subseteq F$ is called an edge cover in $H$ if every vertex in $W$ is incident to an edge in $X$. Given a nonnegative cost function $c: F \rightarrow \mathbf{R}_{+}$, the edge cover problem asks for finding an edge cover $X \subseteq F$ that minimizes the cost $c(X)=\sum_{e \in X} c(e)$. This problem is known to be polynomial time solvable by graph matching (see, e.g., [27, §19.3]). Given a nonnegative submodular function $\rho: 2^{F} \rightarrow \mathbf{R}_{+}$, the submodular edge cover problem asks for finding an edge cover $X \subseteq F$ that minimizes the cost $\rho(X)$. This is a special case of the submodular cost set cover problem with $U=W$ and $N=F$. 
A different type of generalization of the set cover problem was introduced by Hayrapetyan, Swamy, and Tardos [12], in which a submodular penalty cost was imposed. Chudak and Nagano [3] developed an approximation algorithm for this problem using the Lovász extension and the non-smooth convex minimization algorithms of Nesterov [23, 24]. The present paper aims at providing another effective use of the Lovász extension in design of approximation algorithms.

In this paper, we first introduce a natural convex programming relaxation of the submodular vertex cover problem using the Lovász extension and prove that the relaxation problem has a half-integral optimal solution. This extends the result of Nemhauser and Trotter [22] for the vertex cover problem. Accordingly, a rounding algorithm for the vertex cover problem achieves an approximation factor of 2 , and we further show that it can be performed by one application of submodular function minimization on a ring family.

In addition, we describe approximation algorithms for the submodular cost set cover problem. Extending the algorithm of Hochbaum [13], we devise a rounding algorithm based on a convex programming relaxation. We also present a primal-dual algorithm that extends the algorithm of Bar-Yehuda and Even [1]. Both of these algorithms successfully achieve an approximation guarantee of $\eta$.

Alternatively, one can obtain a $k$-approximation solution for the submodular cost set cover problem in a very simple way. Interestingly, we will see that this bound $k$ is an essentially tight bound on the approximability for the submodular cost set cover problem. This will be shown by exhibiting the difficulty of the submodular edge cover problem. Our analysis depends on a technique similar to that of Svitkina and Fleischer [28], and utilizes a celebrated result of Erdős and Rényi [5] on random graphs. We also show that the submodular edge cover problem is NP-hard, whereas the edge cover problem can be solved efficiently by weighted matching algorithms.

The present paper is organized as follows. Section 2 provides preliminaries on submodular functions and associated polyhedra. In Section 3, we give an efficient rounding 2-approximation algorithm for the submodular vertex cover problem. In Section 4, we describe approximation algorithms for the submodular cost set cover problem. Finally, in Section 5, we present hardness results on the submodular edge cover problem.

\section{Submodular Functions and Convexity}

In this section, we provide preliminaries on submodular functions and associated polyhedra.

We denote $N=\{1, \ldots, n\}$. Let $f: 2^{N} \rightarrow \mathbf{R}$ be a set function with $f(\emptyset)=0$. The function $f$ is called nonnegative if $f(X) \geq 0$ for each $X \subseteq N$. The function $f$ is called monotone if $f(X) \leq f(Y)$ for each pair of subsets $X, Y \subseteq N$ with $X \subseteq Y \subseteq N$. Obviously, a monotone function $f$ with $f(\emptyset)=0$ is nonnegative.

Throughout this paper, we assume that $\rho: 2^{N} \rightarrow \mathbf{R}$ is a nonnegative submodular function with $\rho(\emptyset)=0$, which is not necessarily monotone. We also assume that the function $\rho$ is given by a value-giving oracle. Note that the nonnegative submodularity of $\rho$ implies the subadditivity, 
that is, we have $\rho(X)+\rho(Y) \geq \rho(X \cup Y)$ for all $X, Y \subseteq N$. For a vector $z \in \mathbf{R}^{N}$ and a subset $X \subseteq N$, we denote $z(X)=\sum_{i \in X} z(i)$.

Associated with the submodular function $\rho$, we consider a polyhedron

$$
\mathrm{P}(\rho)=\left\{z \mid z \in \mathbf{R}^{N}, z(Y) \leq \rho(Y), \forall Y \subseteq N\right\}
$$

which is called a submodular polyhedron. A vector in $\mathrm{P}(\rho)$ is called a subbase. For any subbase $z$, we say $X \subseteq N$ is $z$-tight if $z(X)=\rho(X)$. The submodularity of $\rho$ implies that for any subbase $z$ the collection of all $z$-tight subsets is closed under union and intersection.

Linear optimization over the submodular polyhedron can be solved efficiently by the greedy algorithm of Edmonds [4]. Given a nonnegative vector $p \in \mathbf{R}_{+}^{N}$, consider a linear ordering $L=\left(i_{1}, \cdots, i_{n}\right)$ such that $p\left(i_{1}\right) \geq p\left(i_{2}\right) \geq \cdots \geq p\left(i_{n}\right)$. For any $i_{j} \in N$, we denote $L\left(i_{j}\right)=$ $\left\{i_{1}, \cdots, i_{j}\right\}$. The greedy algorithm with respect to $L$ generates a vector $z_{L} \in \mathbf{R}^{N}$ determined by

$$
z_{L}(i):=\rho(L(i))-\rho(L(i) \backslash\{i\}) .
$$

Then $z_{L}$ is an extreme point of $\mathrm{P}(\rho)$ maximizing the inner product $\langle p, z\rangle=\sum_{i \in N} p(i) z(i)$ among $z \in \mathrm{P}(\rho)$.

Let $p_{1}>p_{2}>\cdots>p_{m}$ be the distinct values of $p$. For each $j=1, \ldots, m$, we denote $N_{j}=\left\{i \mid p(i) \geq p_{j}\right\}$. We now define $\widehat{\rho}(p)$ by

$$
\widehat{\rho}(p)=\sum_{j=1}^{m}\left(p_{j}-p_{j+1}\right) \rho\left(N_{j}\right),
$$

where $p_{m+1}=0$. Then it follows from the validity of the greedy algorithm that

$$
\widehat{\rho}(p)=\max \{\langle p, z\rangle \mid z \in \mathrm{P}(\rho)\},
$$

which implies the convexity of $\widehat{\rho}$.

Note that the above definition of $\widehat{\rho}$ is free from the submodularity of $\rho$. For a set function $f$ in general, we define $\widehat{f}$ in the same way. Then $\widehat{f}\left(\chi_{X}\right)=f(X)$ holds for any $X \subseteq N$, where $\chi_{X} \in \mathbf{R}^{N}$ is the characteristic vector defined by $\chi_{X}(i)=1$ for $i \in X$ and $\chi_{X}(i)=0$ for $i \in N \backslash X$. Hence we may regard $\widehat{f}$ as an extension of $f$.

The restriction of $\widehat{f}$ to the hypercube $[0,1]^{N}$ can be interpreted as follows. A linear ordering $L$ corresponds to the simplex whose extreme points are given by the characteristic vectors of $L(i)$ for $i \in N$ and the empty set. Since there are $n$ ! linear orderings of $N$, the hypercube $[0,1]^{N}$ can be partitioned into $n$ ! congruent simplices obtained by this way. Determine the function values of $\widehat{f}$ in each simplex by the linear interpolation of the values at the extreme points. The resulting function $\widehat{f}$ is a continuous function on the hypercube.

The following theorem provides a connection between submodularity and convexity.

Theorem 1 (Lovász [19]). A set function $f$ is submodular if and only if $\widehat{f}$ is convex. 


\section{The Submodular Vertex Cover Problem}

In this section, we introduce a natural continuous relaxation of the submodular vertex cover problem using the Lovász extension of $\rho: 2^{V} \rightarrow \mathbf{R}$. We prove that the relaxation has a halfintegral optimal solution and the rounding algorithm achieves an approximation guarantee of 2 for the submodular vertex cover problem. Furthermore, we show that a half-integral optimal solution can be obtained by one execution of submodular function minimization over a ring family.

\subsection{Half-integrality}

We start with the vertex cover problem, which can be formulated as an integer programming problem. The linear programming relaxation is given as follows.

$$
\begin{array}{ll}
(\mathrm{LPR}) \quad \text { Minimize } & \sum_{v \in V} c(v) x(v) \\
\text { subject to } & x(u)+x(v) \geq 1 \quad((u, v) \in E) \\
& x(v) \geq 0 \quad(v \in V) .
\end{array}
$$

Nemhauser and Trotter [22] showed that (LPR) has a half-integral optimal solution. This can be derived from the following lemma in matrix theory.

Lemma 2. Let $A$ be a nonsingular $\{0, \pm 1\}$-matrix each row and each column of which has at most two nonzero entries. Then every entry of the inverse matrix $A^{-1}$ is a half integer.

Proof. We prove this statement by induction on the size of $A$. Suppose that $A$ is an $\ell \times \ell$ matrix and that the statement holds for matrices of smaller size. We call $P A Q$ an admissible transformation if $P$ and $Q$ are nonsingular $\{0, \pm 1\}$-matrices having exactly one nonzero entry in each row and column.

If $A$ has a row with only one nonzero entry, then $A$ can be brought into

$$
\tilde{A}=\left(\begin{array}{ll}
1 & o \\
b & D
\end{array}\right)
$$

by an admissible transformation, where $b$ is a column vector and $o$ is a row vector whose entries are all zero. The inverse of $\tilde{A}$ is given by

$$
\tilde{A}^{-1}=\left(\begin{array}{cc}
1 & o \\
-D^{-1} b & D^{-1}
\end{array}\right) .
$$

By the inductive assumption, $D^{-1}$ is half-integral, and so is $\tilde{A}^{-1}$. Since $A^{-1}$ can be obtained from $\tilde{A}^{-1}$ by an admissible transformation, $A^{-1}$ is also a half-integral matrix.

Similarly, if $A$ has a column with only one nonzero entry, then $A^{-1}$ is a half-integral matrix. 
We now consider the remaining case where every row and column of $A$ has exactly two nonzero entries. By an admissible transformation, $A$ can be turned into a block-diagonal matrix having each diagonal block $B$ in the form of

$$
B=\left(\begin{array}{cccc}
1 & & & 1 \\
1 & 1 & & \\
& \ddots & \ddots & \\
& & 1 & 1
\end{array}\right),
$$

whose inverse matrix is half-integral. Thus $A^{-1}$ is a half-integral matrix.

The half-integrality result on (LPR) naturally leads to an LP-rounding 2-approximation algorithm for the vertex cover problem. Bar-Yehuda and Even [22] developed a primal-dual 2-approximation algorithm that runs in $\mathrm{O}(|E|)$ time.

We now introduce a continuous relaxation $(\mathrm{CPR})$ of the submodular vertex cover problem:

$$
\begin{array}{ll}
(\mathrm{CPR}) \quad \text { Minimize } & \widehat{\rho}(x) \\
\text { subject to } & x(u)+x(v) \geq 1 \quad((u, v) \in E) \\
& x(v) \geq 0 \quad(v \in V) .
\end{array}
$$

This convex programming problem can be solved in polynomial time by the ellipsoid method.

Lemma 3. The relaxation problem (CPR) has a half-integral optimal solution.

Proof. Let $x^{\circ}$ be an optimal solution of (CPR). Consider a linear ordering $L=\left(v_{1}, \ldots, v_{n}\right)$ such that $x^{\circ}\left(v_{1}\right) \geq x^{\circ}\left(v_{2}\right) \geq \cdots \geq x^{\circ}\left(v_{n}\right)$. Then $x^{\circ}$ is an optimal solution to the following linear programming problem.

$$
\begin{aligned}
\text { (SLP) Minimize } & \widehat{\rho}(x) \\
\text { subject to } & x(u)+x(v) \geq 1 \quad((u, v) \in E) \\
& x\left(v_{j}\right)-x\left(v_{j+1}\right) \geq 0 \quad(j=1, \ldots, n-1) \\
& x\left(v_{n}\right) \geq 0
\end{aligned}
$$

Note that the objective function is linear in the feasible region. The coefficient matrix of (SLP) is a $\{0, \pm 1\}$-matrix, each row of which has at most two nonzero entries. By Lemma 2, any nonsingular submatrix has a half-integral inverse matrix. Hence (SLP) has a half-integral optimal solution $x^{*}$, which is also optimal to (CPR).

\subsection{A rounding algorithm}

Let $x^{*}$ be a half-integral optimal solution to (CPR). Then $X^{*}:=\left\{v \mid x^{*}(v) \geq \frac{1}{2}\right\}$ is a vertex cover. The following theorem shows that $X^{*}$ is a 2-approximation solution for the submodular vertex cover problem. 
Theorem 4. The vertex cover $X^{*}$ satisfies $\rho\left(X^{*}\right) \leq 2 \rho(X)$ for any vertex cover $X$ in $G$.

Proof. The half-integral optimal solution $x^{*}$ can be expressed by $x^{*}=\frac{1}{2} \chi_{X^{\prime}}+\frac{1}{2} \chi_{X^{*}}$, where $X^{\prime}:=\{v \mid x(v)=1\}$. Then $\widehat{\rho}\left(x^{*}\right)=\frac{1}{2} \rho\left(X^{\prime}\right)+\frac{1}{2} \rho\left(X^{*}\right) \geq \frac{1}{2} \rho\left(X^{*}\right)$ holds. Since $\rho\left(x^{*}\right)$ is the optimal value of the relaxation problem (CPR), we have $\widehat{\rho}\left(x^{*}\right) \leq \widehat{\rho}\left(\chi_{X}\right)=\rho(X)$ for any vertex cover $X$ in $G$. Therefore, we obtain $\rho\left(X^{*}\right) \leq 2 \widehat{\rho}\left(x^{*}\right) \leq 2 \rho(X)$.

We now discuss a combinatorial algorithm for finding a half-integral optimal solution to the relaxation problem (CRP). Let $V^{+}$and $V^{-}$be the copies of $V$. We denote by $v^{+} \in V^{+}$and $v^{-} \in V^{-}$the copies of $v \in V$. We also denote the copies of $X \subseteq V$ by $X^{+} \subseteq V^{+}$and $X^{-} \subseteq V^{-}$. Construct a bipartite graph $G^{ \pm}=\left(V^{+}, V^{-} ; E^{ \pm}\right)$with vertex sets $V^{+}$and $V^{-}$. The edge set $E^{ \pm}$is given by $E^{ \pm}=\left\{\left(u^{+}, v^{-}\right),\left(v^{+}, u^{-}\right) \mid(u, v) \in E\right\}$. For a vertex cover $\left(X^{+}, Y^{-}\right)$, we define its rank by $\rho(X)+\rho(Y)$. Observe that if $\left(X^{+}, Y^{-}\right)$is a vertex cover, then $\left(X^{+} \cap Y^{+}, X^{-} \cup Y^{-}\right)$ and $\left(X^{+} \cup Y^{+}, X^{-} \cap Y^{-}\right)$are also vertex covers.

Lemma 5. Let $\left(X^{+}, Y^{-}\right)$be a vertex cover in $G^{ \pm}$with minimum rank. Then $x=\frac{1}{2}\left(\chi_{X}+\chi_{Y}\right)$ is a half-integral optimal solution of (CPR).

Proof. For any half-integral feasible solution $x$ of $(\mathrm{CPR})$, we assign a pair of vertex subsets $X=\{v \mid x(v)=1\}$ and $Y=\left\{v \mid x(v) \geq \frac{1}{2}\right\}$. Then $\left(X^{+}, Y^{-}\right)$is a vertex cover in $G^{ \pm}$, and $\widehat{\rho}(x)=\frac{1}{2}[\rho(X)+\rho(Y)]$ holds. Conversely, for any vertex cover $\left(X^{+}, Y^{-}\right)$in $G^{ \pm}, x=\frac{1}{2}\left(\chi_{X}+\chi_{Y}\right)$ is a feasible solution of (CPR), and $\widehat{\rho}(x)=\frac{1}{2}[\rho(X \cap Y)+\rho(X \cup Y)] \leq \frac{1}{2}[\rho(X)+\rho(Y)]$ holds. Therefore, a half-integral optimal solution $x^{*}$ of $(\mathrm{CPR})$ can be obtained by $x^{*}=\frac{1}{2}\left(\chi_{X}+\chi_{Y}\right)$ from a minimum rank vertex cover $\left(X^{+}, Y^{-}\right)$in $G^{ \pm}$.

For a vertex subset $Z \subseteq V$, let $\Gamma(Z)$ denote the set of vertices adjacent to $Z$ in $G$, namely $\Gamma(Z)=\{v \mid \exists u \in Z,(u, v) \in E\}$. For any $X, Y, Z \subseteq V$ with $Z=V \backslash X$, the pair $\left(X^{+}, Y^{-}\right)$ is a vertex cover in $G^{ \pm}$if and only if $\Gamma(Z) \subseteq Y$. We now consider a family $\mathcal{D}$ of subsets $D=Z^{+} \cup Y^{-}$of $V^{+} \cup V^{-}$such that $\Gamma(Z) \subseteq Y$. Then $\mathcal{D}$ forms a ring family, i.e., $\mathcal{D}$ is closed with respect to union and intersection. Note that $\left(X^{+}, Y^{-}\right)$is a vertex cover in $G^{ \pm}$if and only if $X^{+}=V^{+} \backslash D$ and $Y^{-}=D \cap V^{-}$for some $D \in \mathcal{D}$. For each $D=Z^{+} \cup Y^{-}$in $\mathcal{D}$, we assign $f(D):=\rho(V \backslash Z)+\rho(Y)$. Then $f$ is a submodular function on $\mathcal{D}$. Thus finding a minimum rank vertex cover reduces to minimizing the submodular function $f$ on the ring family $\mathcal{D}$. Therefore, by Lemma 5, a half-integral optimal solution of (CPR) can be obtained by one execution of submodular function minimization over a ring family.

\section{The Submodular Cost Set Cover Problem}

In this section, we present approximation algorithms for the submodular cost set cover problem.

For each $u \in U$, we denote $N_{u}=\left\{i \mid u \in S_{i}\right\}$. The maximum frequency $\eta$ is given by $\eta=\max \left\{\left|N_{u}\right| \mid u \in U\right\}$. Note that the special case with $\eta=2$ is essentially the submodular vertex cover problem, for which we have presented a 2-approximation algorithm in $\S 3$. 
For the standard set cover problem (which means $\rho=c$ ), it is known that the greedy algorithm achieves an approximation guarantee of $\mathrm{H}_{k}$, where $\mathrm{H}_{k}=1+\frac{1}{2}+\cdots+\frac{1}{k}=\mathrm{O}(\ln k)$ (see, e.g., [29]). As for the submodular cost set cover problem, the performance of the greedy set cover algorithm is no better than a simple $k$-approximation algorithm of $\S 4.1$. This will be shown in $\S 4.2$.

In contrast, the LP-rounding algorithm of Hochbaum [13] can be extended to achieve the same performance guarantee of the maximum frequence $\eta$ for the submodular cost set cover problem. The resulting algorithm, presented in $\S 4.3$, requires to solve a convex optimization problem by the ellipsoid method. To obviates this part, we also devise a factor $\eta$ primal-dual approximation algorithm in $\S 4.4$ by extending the algorithm of Bar-Yehuda and Even [1].

\subsection{A simple algorithm}

We start with a simple approximation algorithm. For $u \in U$, let $X_{u} \subseteq N$ denote a minimizer of $\rho(X)$ among all the subsets $X \subseteq N$ that covers $u$. Then $X^{\bullet}=\bigcup_{u \in U} X_{u}$ is a set cover.

Proposition 6. The set cover $X^{\bullet}$ satisfies $\rho\left(X^{\bullet}\right) \leq k \rho(X)$ for any set cover $X$.

Proof. Let $X$ be an arbitrary set cover. By the definition of $X_{u}$, we have $\rho\left(X_{u}\right) \leq \rho(X)$ for each $u \in U$. The subadditivity of $\rho$ implies that $\rho\left(X^{\bullet}\right) \leq \sum_{u \in U} \rho\left(X_{u}\right) \leq k \rho(X)$.

For each $u \in U, X_{u}$ can be computed by applying submodular function minimization $\left|N_{u}\right|$ times. Thus, Proposition 6 suggests a strongly polynomial $k$-approximation algorithm for the submodular cost set cover problem.

\subsection{A greedy algorithm}

We naturally generalize the greedy algorithm for the set cover problem. The greedy algorithm for the submodular cost set cover problem keeps a subset $T \subseteq N$. Given $T \subseteq N$, we say an element $i \in N$ is valid if $S_{i} \backslash S_{T} \neq \emptyset$. For $T \subseteq N$ and a valid element $i \in N$, define the cost-effectiveness of $i$ to be $\frac{\rho(T \cup\{i\})-\rho(T)}{\left|S_{i} \backslash S_{T}\right|}$. The algorithm starts with $T:=\emptyset$ and iteratively adds a valid element with the smallest cost-effectiveness to $T$ until $T$ becomes a set cover.

\section{Greedy algorithm for the submodular cost set cover problem}

Step 0: Put $T:=\emptyset$.

Step 1: Repeat the following (1-1) to (1-2) until $T$ covers every element in $U$.

(1-1) Select a valid element $i \in N$ with minimum value of $\frac{\rho(T \cup\{i\})-\rho(T)}{\left|S_{i} \backslash S_{T}\right|}$.

(1-2) Set $T:=T \cup\{i\}$.

Step 2: Return $T$. 
For the standard set cover problem, the above greedy algorithm achieves an approximation guarantee of $\mathrm{H}_{k}=\mathrm{O}(\ln k)$. The following examples of the submodular cost set cover problem show that the performance of the greedy algorithm can be no better or worse than that of the simple $k$-approximation algorithm of $\S 4.1$, even if $\rho$ is monotone.

Example. Suppose $U=\left\{u_{1}, \ldots, u_{n-1}\right\}$ and $\mathcal{S}=\left\{S_{i} \mid i \in N\right\}$ is given by $S_{i}=\left\{u_{i}\right\}$ for each $i \in N \backslash\{n\}$, and $S_{n}=U$. Then the set covers are $\{n\}, N \backslash\{n\}$, and $N$. Consider the submodular cost set cover problem for $\rho$, where $\rho$ is a nonnegative submodular function defined by

$$
\rho(X)=\min \{1,|X|,|N \backslash X|\} \quad(X \subseteq N),
$$

The greedy algorithm returns the subset $\{n\}$ which has a cost of 1 , whereas the optimal value is $\rho(N)=0$. Next, we suppose that $\rho$ is a monotone submodular function defined by

$$
\rho(X)=\min \{1,|X \backslash\{n\}|\}+(k-\epsilon) \cdot \min \{1,|X \cap\{n\}|\} \quad(X \subseteq N),
$$

where $k=|U|=n-1$. The greedy algorithm returns the subset $\{n\}$ which has a cost of $k-\epsilon$, whereas the optimal value is $\rho(N \backslash\{n\})=1$.

\subsection{A rounding algorithm}

Consider a convex programming relaxation of the submodular cost set cover problem:

$$
\begin{array}{ll}
\text { (SCP) Minimize } & \widehat{\rho}(x) \\
\text { subject to } & \sum_{i \in N_{u}} x(i) \geq 1 \quad(u \in U) \\
& x(i) \geq 0 \quad(i \in N) .
\end{array}
$$

This problem can be solved in polynomial time with the aid of the ellipsoid method.

Let $\rho^{\circ}: 2^{N} \rightarrow \mathbf{R}$ be defined by

$$
\rho^{\circ}(X)=\min \{\rho(Z) \mid X \subseteq Z \subseteq N\} \quad(X \subseteq N) .
$$

Clearly, $\rho^{\circ}$ is monotone. It is known that $\rho^{\circ}$ is submodular (see, e.g., [8, Section 3.1(b)]). By definition, we have $\rho(X) \geq \rho^{\circ}(X)$ for all $X \subseteq N$. Therefore, for all $x \in \mathbf{R}_{+}^{N}$ we have $\widehat{\rho}(x) \geq$ $\hat{\rho}^{\circ}(x)$. For each $X \subseteq N$, let $X^{\circ}$ denote the unique minimal subset $Z$ such that $X \subseteq Z \subseteq N$ and $\rho(Z)=\rho^{\circ}(X)$. Then $\rho^{\circ}(X)=\rho\left(X^{\circ}\right)$ holds for any $X \subseteq N$.

Let $x^{*} \in \mathbf{R}^{N}$ be an optimal solution to (SCP). Then $T=\left\{i \mid x^{*}(i) \geq 1 / \eta\right\}$ is a set cover, and so is $T^{\circ}$. Note that $T^{\circ}$ can be obtained by executing submodular function minimization. The following theorem shows that $T^{\circ}$ is an $\eta$-approximate solution for the submodular cost set cover problem.

Theorem 7. The set cover $T^{\circ}$ satisfies $\rho\left(T^{\circ}\right) \leq \eta \rho(X)$ for any set cover $X$. 
Proof. Since $\widehat{\rho}\left(x^{*}\right)$ is the optimal value of the relaxation problem (SCP), we have $\widehat{\rho}^{\circ}\left(x^{*}\right) \leq$ $\widehat{\rho}\left(x^{*}\right) \leq \rho\left(\chi_{X}\right)=\rho(X)$ for any set cover $X$. The function $\widehat{\rho}^{\circ}$ is monotone and positively homogeneous. Then it follows from $\eta x^{*} \geq \chi_{T}$ that $\eta \widehat{\rho}^{\circ}\left(x^{*}\right)=\widehat{\rho}^{\circ}\left(\eta x^{*}\right) \geq \widehat{\rho}^{\circ}\left(\chi_{T}\right)=\rho^{\circ}(T)=$ $\rho\left(T^{\circ}\right)$. Thus, we obtain $\rho\left(T^{\circ}\right) \leq \eta \rho(X)$.

\subsection{A primal-dual algorithm}

We now present a primal-dual algorithm using the relaxation problem (SCP). Given a vector $x \in \mathbf{R}_{+}^{V}$, we have $\widehat{\rho}(x)=\max \{\langle x, z\rangle \mid z \in \mathrm{P}(\rho)\}$. Thus, the value $\widehat{\rho}(x)$ is equal to the optimal value of the following dual problem with variables $\xi(X)$ for all nonempty $X \subseteq N$.

$$
\begin{array}{cl}
\text { Minimize } & \sum_{X \subseteq N} \rho(X) \cdot \xi(X) \\
\text { subject to } & \sum_{X: i \in X \subseteq N} \xi(X)=x(i) \quad(i \in N) \\
& \xi(X) \geq 0 \quad(X \subseteq N) .
\end{array}
$$

Therefore, the problem (SCP) can be written as a linear program:

$$
\begin{array}{ll}
\text { Minimize } & \sum_{X \subseteq N} \rho(X) \cdot \xi(X) \\
\text { subject to } & \sum_{i \in N_{u}} x(i) \geq 1 \quad(u \in U) \\
& \sum_{X: i \in X \subseteq N} \xi(X)=x(i) \quad(i \in N) \\
& \xi(X) \geq 0 \quad(X \subseteq N) .
\end{array}
$$

Here, we neglect the redundant nonnegativity constraint of $x(i)$ for $i \in N$. Therefore, the dual problem to $(\mathrm{SCP})$ is given as follows.

$$
\begin{array}{ll}
\text { (DCP) Maximize } & \sum_{u \in U} y(u) \\
\text { subject to } & z \in \mathrm{P}(\rho), \\
& \sum_{u \in S_{i}} y(u)=z(i) \quad(i \in N), \\
& y(u) \geq 0 \quad(u \in U) .
\end{array}
$$

The primal-dual algorithm keeps a feasible solution $(y, z)$ of (DCP) and a subset $T \subseteq N$ that is $z$-tight. The algorithm starts with $y:=0, z:=0$ and $T:=\emptyset$. Since $\rho$ is a nonnegative submodular function with $\rho(\emptyset)=0$, this gives a feasible solution of (DCP) and we have $z(T)=$ $\rho(T)$. While $T$ is not a set cover, there must be an element $u \in U$ which is not covered by $T$. The algorithm augments $y(u)$ and $z(i)$ for $i \in N_{u}$ as much as possible without violating the constraints in (DCP). Then the algorithm updates $T$ to be the unique maximal set with 
$z(T)=\rho(T)$. The algorithm iterates this procedure until $T$ becomes a set cover. The algorithm is now described more precisely as follows.

\section{Primal-dual algorithm for the submodular cost set cover problem}

Step 0: Put $y:=0, z:=0$, and $T:=\emptyset$.

Step 1: Repeat the following (1-1) to (1-4) until $T$ covers all elements of $U$.

(1-1) Select an element $u \in U \backslash S_{T}$ and put $Y:=N_{u}$.

(1-2) Compute $\alpha:=\max \left\{\lambda \mid z+\lambda \chi_{Y} \in \mathrm{P}(\rho)\right\}$.

(1-3) Put $y(u):=y(u)+\alpha$ and $z:=z+\alpha \chi_{Y}$.

(1-4) Update $T$ to be the unique maximal set with $z(T)=\rho(T)$.

\section{Step 2: Return $T$.}

It is easy to see that the primal-dual algorithm indeed keeps a feasible solution of (DCP) and a $z$-tight set $T \subseteq N$. We now analyze the running time of the primal-dual algorithm. Since

$$
z+\lambda \chi_{Y} \in \mathrm{P}(\rho) \text { if and only if } \lambda|X \cap Y| \leq \rho(X)-z(X)(X \subseteq N),
$$

the computation of $\alpha$ in Step (1-2) is tantamount to minimizing $[\rho(X)-z(X)] /|X \cap Y|$ subject to $X \cap Y \neq \emptyset$. This minimization problem can be solved by the Newton method within the same running time as submodular function minimization [7,21]. The obtained minimizer $X$ satisfies $\rho(X)=z(X)+\alpha|X \cap Y|$ and $|X \cap Y| \geq 1$. Note that $\rho(T)=z(T)$ and $|T \cap Y|=0$. Then, after the subsequent update of $z$ in Step (1-3), it holds that $z(X)=\rho(X)$ and $z(T)=\rho(T)$, which implies $z(X \cup T)=\rho(X \cup T)$ by the submodularity of $\rho$. Therefore, $T$ gets larger as a result of Step (1-4). Thus, the algorithm terminates after at most $n$ iterations.

We now analyze the approximation ratio of the primal-dual algorithm. The following theorem shows that the primal-dual algorithm is an $\eta$-approximation algorithm.

Theorem 8. At the termination of the primal-dual algorithm, $\rho(T) \leq \eta \rho(X)$ holds for any set cover $X \subseteq N$, where $\eta$ is the maximum frequency.

Proof. By the definition of $\eta$, for any set cover $X$, we have

$$
\sum_{u \in U} y(u) \leq \sum_{i \in X} \sum_{u \in S_{i}} y(u) \leq \eta \sum_{u \in U} y(u) .
$$

Since $T \subseteq N$ is a set cover with $z(T)=\rho(T)$, it follows from the feasibility of $(y, z)$ in (DCP) that

$$
\rho(T)=z(T)=\sum_{i \in T} \sum_{u \in S_{i}} y(u) \leq \eta \sum_{u \in U} y(u) .
$$

On the other side, for any set cover $X \subseteq N$, we have

$$
\rho(X) \geq z(X)=\sum_{i \in X} \sum_{u \in S_{i}} y(u) \geq \sum_{u \in U} y(u) .
$$

Thus we obtain $\rho(T) \leq \eta \rho(X)$ for any set cover $X$. 


\section{The Submodular Edge Cover Problem}

This section is devoted to hardness results on the submodular edge cover problems. We first show that the problem is NP-hard in $\S 5.1$, and then discuss the inapproximability in $\S 5.2$.

\subsection{Intractability}

The edge cover problem is solvable in polynomial time by weighted mathcing algorithms. In contrast, we now show that the submodular edge cover problem is NP-hard. For this purpose, it is convenient to consider the following two problems.

MIN 2-SAT: Let $\mathcal{X}=\left\{x_{1}, \ldots, x_{n}\right\}$ be the set of Boolean variables and let $\mathcal{C}=\left\{C_{1}, \ldots, C_{m}\right\}$ be the set of clauses, each of which is in the form of $z_{1} \vee z_{2}$, where each $z_{j}$ is a variable $x_{\ell}$ or its negation $\bar{x}_{\ell}$. The MIN 2-SAT problem asks for finding a truth assignment $\tau: \mathcal{X} \rightarrow\{$ True, False $\}$ that minimizes the number of satisfied clauses.

Switching Submodular Function Minimization (SSFM): We denote $[n]=\{1, \ldots, n\}$ and $[\bar{n}]=\{\overline{1}, \ldots, \bar{n}\}$. Define $\bar{X}:=\{\bar{i} \mid i \in X\}$ for each $X \subseteq[n]$. Given a submodular set function $f: 2^{[n] \cup[\bar{n}]} \rightarrow \mathbf{R}$, the switching submodular function minimization problem asks for finding a bipartition $(X, Y)$ of $[n]$ that minimizes $f(X \cup \bar{Y})$.

The MIN 2-SAT problem is known to be NP-hard [17]. First, we show that the MIN 2-SAT problem is a special case of the SSFM problem. Next, we show that the SSFM problem is a special case of the submodular edge cover problem. Thus, we conclude that the submodular edge cover problem is NP-hard.

Proposition 9. The switching submodular function minimization problem is NP-hard.

Proof. We convert the MIN 2-SAT problem into the form of the SSFM problem. Let $\mathcal{X}=$ $\left\{x_{1}, \ldots, x_{n}\right\}$ be the set of Boolean variables and $\mathcal{C}=\left\{C_{1}, \ldots, C_{m}\right\}$ be the set of clauses in an arbitrary instance of MIN 2-SAT. Given a truth assignment $\tau: \mathcal{X} \rightarrow\{$ True, False\}, we denote $Z_{\tau}=\left\{i \mid x_{i}=\right.$ True $\} \cup\left\{\bar{i} \mid x_{i}=\right.$ False $\}$. Note that $\left\{Z_{\tau} \mid \tau\right.$ is a truth assignment $\}=$ $\{X \cup([\bar{n}] \backslash \bar{X}) \mid X \subseteq[n]\}$.

Consider a complete digraph $K=([n] \cup[\bar{n}], A)$ with vertex set $[n] \cup[\bar{n}]$ and arc set $A$. Let $\sigma:\left\{x_{1}, \ldots, x_{n}, \bar{x}_{1}, \ldots, \bar{x}_{n}\right\} \rightarrow[n] \cup[\bar{n}]$ be a mapping such that $\sigma\left(x_{i}\right)=i$ and $\sigma\left(\bar{x}_{i}\right)=\bar{i}$ for each $i=1, \ldots, n$. We define the capacity $q \in \mathbf{R}^{A}$ as follows. For each clause $C_{j}=y \vee z$, we let $A_{j}=\{\sigma(y) \sigma(z), \sigma(z) \sigma(y), \sigma(y) \sigma(\bar{y}), \sigma(z) \sigma(\bar{z})\}$ and define the partial capacity $q_{j} \in \mathbf{R}_{+}^{A}$ by

$$
q_{j}(a)=\left\{\begin{array}{cl}
1 / 2 & \text { if } a \in A_{j}, \\
0 & \text { if } a \in A \backslash A_{j} .
\end{array}\right.
$$


Let $\kappa_{j}: 2^{[n] \cup[\bar{n}]} \rightarrow \mathbf{R}$ be a cut function with respect to $K$ and $q_{j}$. It is easy to observe that for any truth assignment $\tau: \mathcal{X} \rightarrow\{$ True, False $\}$,

$$
\begin{aligned}
& \kappa_{j}\left(Z_{\tau}\right)=1 \text { if and only if } C_{j} \text { is satisfied, } \\
& \kappa_{j}\left(Z_{\tau}\right)=0 \text { if and only if } C_{j} \text { is not satisfied. }
\end{aligned}
$$

Define the total capacity $q \in \mathbf{R}_{+}^{A}$ and the function $\kappa: 2^{[n] \cup[\bar{n}]} \rightarrow \mathbf{R}$ by $q:=\sum_{j=1}^{m} q_{j}$ and $\kappa:=\sum_{j=1}^{m} \kappa_{j}$, respectively. Then $\kappa$ is a cut function with respect to $K$ and $q$, and hence it is submodular. Furthermore, for any truth assignment $\tau: \mathcal{X} \rightarrow\{$ True, False $\}$, the value $\kappa\left(Z_{\tau}\right)$ is equal to the number of satisfied clauses. Thus, the MIN 2-SAT problem is equivalent to the problem of finding a truth assignment $\tau$ that minimizes $\kappa\left(Z_{\tau}\right)$, which is of the form of the SSFM problem. As the MIN 2-SAT problem is NP-hard [17], the SSFM problem is also NP-hard.

The following lemma assures that the SSFM problem reduces to the one in which the submodular function is strictly monotone.

Lemma 10. Suppose $N=[n] \cup[\bar{n}]$. Given a submodular function $f: 2^{N} \rightarrow \mathbf{R}$, let $f_{\mathrm{m}}: 2^{N} \rightarrow \mathbf{R}$ be a function defined by

$$
f_{\mathrm{m}}(Z)=f(Z)+\delta|Z| \quad(Z \subseteq N),
$$

where $\delta>\max _{i \in N}|f(N \backslash\{i\})-f(N)|$. Then, $f_{\mathrm{m}}$ is a strictly monotone submodular function, and $f_{\mathrm{m}}(X \cup \bar{Y})=f(X \cup \bar{Y})+n \delta$ holds for any bipartition $(X, Y)$ of $[n]$.

Proof. The submodularity of $f_{\mathrm{m}}$ is straightforward. For each $Z \subseteq N$ and each $i \in Z$, we have

$$
f_{\mathrm{m}}(Z)-f_{\mathrm{m}}(Z \backslash\{i\})=\delta+f(Z)-f(Z \backslash\{i\}) \geq \delta+f(N)-f(N \backslash\{i\})>0 .
$$

Therefore, $f_{\mathrm{m}}$ is strictly monotone. The latter assertion is obvious from the definition of $f_{\mathrm{m}}$.

Theorem 11. The submodular edge cover problem is NP-hard.

Proof. By Proposition 9, it suffices to show that the SSFM problem can be reduced to the submodular edge cover problem. Let $f: 2^{[n] \cup[\bar{n}]} \rightarrow \mathbf{R}$ be the submodular set function in an arbitrary instance of the SSFM problem. By Lemma 10, we can assume w.l.o.g. that $f$ is strictly monotone. Let $\left[n^{\circ}\right]=\left\{1^{\circ}, \ldots, n^{\circ}\right\}$. Consider a graph $H=(W, F)$ with edge set $F=[n] \cup[\bar{n}] \cup\left[n^{\circ}\right]$ such that $\left\{1, \overline{1}, 1^{\circ}\right\}, \ldots,\left\{n, \bar{n}, n^{\circ}\right\}$ are vertex disjoint triangles. The function $\rho: 2^{F} \rightarrow \mathbf{R}$ defined by $\rho(Z)=f(Z \cap([n] \cup[\bar{n}]))(Z \subseteq F)$ is monotone and submodular. Let $Z^{*} \subseteq F$ be an edge cover $Z \subseteq F$ that minimizes $\rho(Z)$. Since $f: 2^{[n] \cup[\bar{n}]} \rightarrow \mathbf{R}$ is strictly monotone, we have $\left[n^{\circ}\right] \subseteq Z^{*}$ and $\left|Z^{*} \cap\{i, \bar{i}\}\right|=1$ for each $i \in[n]$. Thus, there exists a bipartition $\left(X^{*}, Y^{*}\right)$ of $[n]$ such that $Z^{*}=X^{*} \cup \bar{Y}^{*} \cup\left[n^{\circ}\right]$. Conversely, for any bipartition $(X, Y)$ of $[n]$, the subset $X \cup \bar{Y} \cup\left[n^{\circ}\right] \subseteq F$ is an edge cover. Therefore, $Z^{*} \cap([n] \cup[\bar{n}])$ is an optimal solution to the SSFM problem. Hence, the SSFM problem is a special case of the submodular edge cover problem. 


\section{$5.2 \quad$ Inapproximability}

In this section, we examine the inapproximability of the submodular edge cover problem. Our analysis is based on a framework similar to that of Svitkina and Fleischer [28] for lower bounds on submodular sparsest cut problems, and uses a sophisticated result on random graphs.

The simple algorithm of $\S 4.1$ achieves an approximation guarantee of $k$ for general submodular cost set cover problems. We will see that this factor is essentially optimal even for the submodular edge cover problem with monotone submodular cost functions. The following theorem is the main result of this section.

Theorem 12. Let $\varepsilon>0$ be any positive real number. In the value-giving oracle model, there is no $\mathrm{O}\left(|W|^{1-\varepsilon}\right)$-approximation algorithm with polynomial number of oracle calls for the submodular edge cover problem on a graph $H=(W, F)$. More precisely, the submodular edge cover problem cannot be approximated within a factor of $o\left(|W| / \ln ^{2}|W|\right)$.

This result immediately implies that the submodular cost set cover problem cannot be approximated within a factor of $o\left(k / \ln ^{2} k\right)$. The proof of Theorem 12 will be given below. The following lemma of [28] is used for obtaining the inapproximability result.

Lemma 13 ([28, Lemma 2.1]). Let $f_{1}$ and $f_{2}$ be two functions defined on $2^{N}$, where $f_{2}$ is parametrized by a string of random bits $R$ but $f_{1}$ is not. Suppose that for any subset $X \subseteq N$, chosen without knowing $R$, the probability over $R$ that $f_{1}(X) \neq f_{2}(X)$ is $n^{-\omega(1)}$. Then, any algorithm that calls a value-giving oracle a polynomial number of times can find a subset $X^{*} \subseteq N$ such that $f_{1}\left(X^{*}\right) \neq f_{2}\left(X^{*}\right)$ with probability at most $n^{-\omega(1)}$.

To prove Theorem 12, we will give a graph $H=(W, F)$ and two monotone submodular functions $\rho_{1}$ and $\rho_{2}$ defined on $2^{F}$ such that

- The function $\rho_{2}$ is parametrized by a random subset $R \subseteq F$ but $\rho_{1}$ is not.

- Without knowledge of $R$, it is difficult to find $X \subseteq F$ such that $\rho_{1}(X) \neq \rho_{2}(X)$.

- It holds that $\mathrm{OPT}_{1}=\Omega(|W|)$ and $\mathrm{OPT}_{2}=\mathrm{O}\left(\ln ^{2}|W|\right)$ with probability at least $3 / 4$, where $\mathrm{OPT}_{i}$ is the optimal value of the submodular edge cover problem for $H$ and $\rho_{i}$ for each $i=1,2$.

Then, the existence of a factor $o\left(|W| / \ln ^{2}|W|\right)$ algorithm would lead to a contradiction.

\section{A random subgraph}

Let $k$ be an even number, and let $H=(W, F)$ be a complete graph with $|W|=k$. The edge set $F$ is of cardinality $n=\frac{1}{2} k(k-1)$. If $X \subseteq F$ is a perfect matching in $H$, then $X \subseteq F$ satisfies the edge cover constraint with respect to $H$.

Let $R \subseteq F$ be a random subset for which each $e \in F$ is chosen independently with an identical probability $\pi \in[0,1]$, where $\pi$ is the parameter that will be defined below. We show some properties of a random subgraph $H_{\pi}=(W, R)$. Denote $\mu=\mathbf{E}[|R|]=\frac{1}{2} k(k-1) \pi$. 
The parameter $\pi$ will be defined so that $R$ contains a perfect matching with high probability. Erdős and Rényi proved the following result on random graphs (cf. [2, Theorem VII.14]).

Theorem 14 (Erdös and Rényi [5]). If $\pi \geq(\ln k+3 \ln \ln k) / 2 k$, then the probability (over the choice of $R$ ) that $H_{\pi}=(W, R)$ does not have a perfect matching is o(1).

In order to evaluate the cardinality of the random subset $R$, we need to know the tail distribution of the sum of Bernoulli trials. The following well-known bound is referred to as a Chernoff bound.

Lemma 15 (Chernoff bounds (see, e.g., [20])). Let $\beta_{1}, \ldots, \beta_{m}$ be independent random variables such that $\operatorname{Pr}\left(\beta_{i}=1\right)=\pi$ and $\operatorname{Pr}\left(\beta_{i}=0\right)=1-\pi$. Let $\beta=\sum_{i=1}^{m} \beta_{i}$ and $\mu_{\beta}=\mathbf{E}[\beta]=$ $\pi m$. For $\alpha \geq 8 \mu_{\beta}$, we have $\operatorname{Pr}(\beta \geq \alpha) \leq \exp (-\alpha)$.

Proof. Set $r=\alpha / \mu_{\beta}$. For each $i=1, \ldots, m$, we have

$$
\mathbf{E}\left[r^{\beta_{i}}\right]=\pi r^{1}+(1-\pi) r^{0}=1+\pi(r-1) \leq \exp (\pi(r-1)) \leq \exp (\pi r) .
$$

Since $\beta_{1}, \ldots, \beta_{m}$ are independent, $r^{\beta_{1}}, \ldots, r^{\beta_{m}}$ are also independent. Hence,

$$
\mathbf{E}\left[r^{\beta}\right]=\prod_{i=1}^{m} \mathbf{E}\left[r^{\beta_{i}}\right] \leq \prod_{i=1}^{m} \exp (\pi r)=\exp \left(\mu_{\beta} r\right)=\exp (\alpha) .
$$

For $\alpha \geq 8 \mu_{\beta}, r=\alpha / \mu_{\beta} \geq \mathrm{e}^{2}$. Using Markov's inequality and (3), for $\alpha \geq 8 \mu_{\beta}$

$$
\operatorname{Pr}(\beta \geq \alpha)=\operatorname{Pr}\left(r^{\beta} \geq r^{\alpha}\right) \leq \frac{\mathbf{E}\left[r^{\beta}\right]}{r^{\alpha}} \leq \frac{\exp (\alpha)}{r^{\alpha}}=\left(\frac{\mathrm{e}}{r}\right)^{\alpha} \leq \exp (-\alpha),
$$

as desired.

Now, we set $\pi=\ln k / k$. Theorem 14 implies that there exists an integer $k_{0}$ such that

$$
\operatorname{Pr}\left(H_{\pi}=(W, R) \text { has a perfect matching }\right)>\frac{3}{4}, \text { for all } k \geq k_{0} .
$$

Since $\mu=\mathbf{E}[|R|]=\frac{1}{2}(k-1) \ln k$, we see from Lemma 15 that

$$
\operatorname{Pr}(|R| \geq 8 \mu) \leq \exp (-8 \mu)=\exp (-4(k-1) \ln k)=k^{-4(k-1)} .
$$

\section{Comparison of two submodular functions}

Consider the following two set functions defined on $2^{F}$ :

$$
\begin{aligned}
& \rho_{1}(X)=\min \{\mu,|X|\} \quad(X \subseteq F), \\
& \rho_{2}(X)=\min \left\{\mu,|X \backslash R|+\min \left\{36 \ln ^{2} k,|X \cap R|\right\}\right\} \quad(X \subseteq F) .
\end{aligned}
$$

The function $\rho_{2}$, but not $\rho_{1}$, is parametrized by the random subset $R$. Regardless of the choice of $R, \rho_{2}(X) \leq \rho_{1}(X)$ for all $X \subseteq F$, and both $\rho_{1}$ and $\rho_{2}$ are monotone submodular functions. Let $\mathrm{OPT}_{i}$ denote the optimal value of the monotone submodular edge cover problem for $H=(W, F)$ and $\rho_{i}$ for $i=1,2$. We now evaluate the gap between $\mathrm{OPT}_{1}$ and $\mathrm{OPT}_{2}$, which plays an important role to prove Theorem 12 . 
Lemma 16. If $H_{\pi}=(W, R)$ has a perfect matching, it holds that $\mathrm{OPT}_{1}=\Omega(k)$ and $\mathrm{OPT}_{2}=$ $\mathrm{O}\left(\ln ^{2} k\right)$.

Proof. For any edge cover $X \subseteq F$, we have $|X| \geq k / 2$. Since $\mu=\frac{1}{2}(k-1) \ln k$, we have $\mathrm{OPT}_{1} \geq \min \{\mu, k / 2\}=\Omega(k)$. Consider the case where $H_{\pi}=(W, R)$ has a perfect matching $X$. Since $X \subseteq R$, we have $\mathrm{OPT}_{2} \leq \rho_{2}(X)=\min \left\{\mu, 36 \ln ^{2} k, k / 2\right\}=\mathrm{O}\left(\ln ^{2} k\right)$.

Note that the probability (over the choice of $R$ ) that $H_{\pi}$ has a perfect matching is at least $\frac{3}{4}$ for a sufficiently large $k$.

The other crucial element towards the proof of Theorem 12 is that, for any fixed $X \subseteq V$, the probability (over the choice of $R$ ) that $\rho_{1}(X) \neq \rho_{2}(X)$ is quite small.

Lemma 17. Fix any subset $X \subseteq F$. Let $R$ be a random subset of $F$ for which each $e \in F$ is chosen independently with probability $\pi=\ln k / k$. Then, the probability (over the choice of $R$ ) that $\rho_{1}(X) \neq \rho_{2}(X)$ is at most $k^{-\omega(1)}$.

Proof. To show the assertion, we consider the case that $|X| \geq 9 \mu$ and the case that $|X| \leq 9 \mu$, separately. We assume that $k$ is sufficiently large.

(i) Suppose that $|X| \geq 9 \mu$. Then, $|R| \leq 8 \mu$ implies $\mu \leq|X \backslash R|$. Furthermore, $\mu \leq|X \backslash R|$ implies $\rho_{1}(X)=\rho_{2}(X)=\mu$. Thus, in view of (4), we obtain

$$
\operatorname{Pr}\left(\rho_{1}(X) \neq \rho_{2}(X)\right) \leq \operatorname{Pr}(|R|>8 \mu) \leq k^{-4(k-1)}=k^{-\omega(1)} .
$$

(ii) Suppose that $|X| \leq 9 \mu$. By the definitions of $\rho_{1}$ and $\rho_{2}, \rho_{1}(X) \neq \rho_{2}(X)$ implies $|X \cap R|>$ $36 \ln ^{2} k$. Thus,

$$
\operatorname{Pr}\left(\rho_{1}(X) \neq \rho_{2}(X)\right) \leq \operatorname{Pr}\left(|X \cap R|>36 \ln ^{2} k\right) .
$$

Clearly, the right hand side of inequality (5) is maximized with respect to $X$ when $|X|=\lfloor 9 \mu\rfloor$. Let $T$ be an arbitrary subset of $F$ such that $|T|=\lfloor 9 \mu\rfloor$ and let $\mu^{\prime}=\mathbf{E}(|T \cap R|)=\pi \cdot\lfloor 9 \mu\rfloor$. Since $9 \mu \pi=\frac{9}{2} \frac{k-1}{k} \ln ^{2} k$, we have $4 \ln ^{2} k \leq \mu^{\prime} \leq \frac{9}{2} \ln ^{2} k$. Lemma 15 implies

$$
\begin{aligned}
\operatorname{Pr}\left(|T \cap R|>36 \ln ^{2} k\right) & =\operatorname{Pr}\left(|T \cap R|>8 \cdot\left(\frac{9}{2} \ln ^{2} k\right)\right) \leq \operatorname{Pr}\left(|T \cap R|>8 \mu^{\prime}\right) \leq \exp \left(-8 \mu^{\prime}\right) \\
& \leq \exp \left(-32 \ln ^{2} k\right) .
\end{aligned}
$$

Hence, for any subset $X \subseteq F$ with $|X| \leq 9 \mu$, we have

$$
\operatorname{Pr}\left(|X \cap R|>36 \ln ^{2} k\right) \leq \operatorname{Pr}\left(|T \cap R|>36 \ln ^{2} k\right) \leq k^{-32 \ln k}=k^{-\omega(1)} .
$$

By (5) and (6), we have $\operatorname{Pr}\left(\rho_{1}(X) \neq \rho_{2}(X)\right) \leq k^{-\omega(1)}$, completing the proof.

Using Lemmas 13 and 17, we obtain the following.

Corollary 18. For any algorithm that calls a polynomial number of value-giving oracle, the probability (over the choice of $R$ ) that it can find a subset $X \subseteq F$ such that $\rho_{1}(X) \neq \rho_{2}(X)$ is at most $k^{-\omega(1)}$. 


\section{Proof of the inapproximability}

Finally, we give a proof of Theorem 12 .

Proof of Theorem 12: Let $k=|W|$. Assume, to the contrary, that there is a polynomial $\gamma$-approximation algorithm $\mathcal{A}$ for the submodular edge cover problem, where $\gamma=o\left(k / \ln ^{2} k\right)$, which succeeds with high probability. Then, we can suppose w.l.o.g. that $\mathcal{A}$ succeeds with probability at least $3 / 4$.

We suppose that $k$ is sufficiently large. Apply the algorithm $\mathcal{A}$ to the submodular edge cover problem for $\rho_{2}$ and $H$, and let $X$ be an edge cover given by $\mathcal{A}$. We only consider the case where $\mathcal{A}$ succeeds and $H_{\pi}$ has a perfect matching, which occurs with probability at least $1-\left(1-\frac{3}{4}\right)-\left(1-\frac{3}{4}\right)=\frac{1}{2}$. It follows from Lemma 16 that $\rho_{1}(X) \geq \mathrm{OPT}_{1}=\Omega(k)$ and $\rho_{2}(X) \leq \gamma \cdot \mathrm{OPT}_{2}=\mathrm{O}\left(\ln ^{2} k \cdot \gamma\right)=o(k)$. As a result, we obtain $\rho_{1}(X) \neq \rho_{2}(X)$ with probability at least $1 / 2$, which contradicts Corollary 18 .

\section{Acknowledgements}

The authors thank Magnús Halldórsson and Lex Schrijver for their helpful comments.

\section{References}

[1] R. Bar-Yehuda and S. Even: A linear time approximation algorithm for the weighted vertex cover problem. J. Algorithms, 2 (1981), pp. 198-203.

[2] B. Bollobás: Random Graphs. Academic Press, 1985.

[3] F. Chudak and K. Nagano: Efficient solutions to relaxations of combinatorial problems with submodular penalties via the Lovász extension and non-smooth convex optimization. Proceedings of the 18th Annual ACM-SIAM Symposium on Discrete Algorithms (2007), pp. $79-88$.

[4] J. Edmonds: Submodular functions, matroids, and certain polyhedra. Combinatorial Structures and Their Applications, R. Guy, H. Hanani, N. Sauer, and J. Schönheim, eds., Gordon and Breach, 1970, pp. 69-87.

[5] P. Erdős and A. Rényi: On the existence of a factor of degree one of a connected random graph. Acta Math. Acad. Sci. Hungar., 17 (1966), pp. 359-368.

[6] U. Feige, V. Mirrokni, and J. Vondrák: Maximizing non-monotone submodular functions. Proceedings of the 48th Annual IEEE Symposium on Foundations of Computer Science (2007), pp. 461-471. 
[7] L. Fleischer and S. Iwata: A push-relabel framework for submodular function minimization and applications to parametric optimization. Discrete Appl. Math., 131 (2003), pp. 311322 .

[8] S. Fujishige: Submodular Functions and Optimization. Elsevier, Amsterdam, 2005.

[9] M. X. Goemans and V. S. Ramakrishnan: Minimizing submodular functions over families of sets. Combinatorica, 15 (1995), pp. 499-513.

[10] M. Grötschel, L. Lovász, and A. Schrijver: The ellipsoid method and its consequences in combinatorial optimization. Combinatorica, 1 (1981), pp. 169-197.

[11] M. Grötschel, L. Lovász, and A. Schrijver: Geometric Algorithms and Combinatorial Optimization. Springer-Verlag, Berlin, 1988.

[12] A. Hayrapetyan, C. Swamy, and É. Tardos: Network design for information networks. Proceedings of the 16th Annual ACM-SIAM Symposium on Discrete Algorithms (2005), pp. $933-942$.

[13] D. S. Hochbaum: Approximation algorithms for the set covering and vertex cover problems. SIAM J. Comput., 11 (1982), pp. 555-556.

[14] S. Iwata: A faster scaling algorithm for minimizing submodular functions. SIAM J. Comput., 32 (2003), pp. 833-840.

[15] S. Iwata, L. Fleischer, and S. Fujishige: A combinatorial strongly polynomial algorithm for minimizing submodular functions. Journal of the ACM, 48 (2001), pp. 761-777.

[16] S. Iwata and J. B. Orlin: A simple combinatorial algorithm for submodular function minimization. Proceedings of the 20th Annual ACM-SIAM Symposium on Discrete Algorithms (2009), pp. 1230-1237.

[17] R. Kohli, R. Krishnamurti, and P. Mirchandani: The minimum satisfiability problem. SIAM J. Discrete Math., 7 (1994), pp. 275-283.

[18] J. Lee, V. Mirrokni, V. Nagarajan, and M. Sviridenko: Maximizing non-monotone submodular functions under matroid and knapsack constraints. Proceedings of the 41th Annual ACM Symposium on Theory of Computing (2009), to appear.

[19] L. Lovász: Submodular functions and convexity. Mathematical Programming — The State of the Art. A. Bachem, M. Grötschel and B. Korte, eds., Springer-Verlag, 1983, 235-257.

[20] M. Mitzenmacher and E. Upfal: Probability and Computing: Randomized Algorithms and Probabilistic Analysis. Cambridge University Press, 2005. 
[21] K. Nagano: A faster parametric submodular function minimization algorithm and applications. METR 2007-43, University of Tokyo, July 2007.

[22] G. L. Nemhauser and L. E. Trotter, Jr.: Properties of vertex packing and independence system polyhedra. Math. Programming, 6 (1974), pp. 48-61.

[23] Y. Nesterov: Smooth minimization of non-smooth functions. Math. Progrmming, 103 (2005), pp. 127-152.

[24] Y. Nesterov: Excessive gap technique in nonsmooth convex minimization. SIAM J. Optim., 16 (2005), pp. 235-249.

[25] J. B. Orlin: A faster strongly polynomial time algorithm for submodular function minimization. Math. Programming, 118 (2009), pp. 237-251.

[26] A. Schrijver: A combinatorial algorithm minimizing submodular functions in strongly polynomial time. J. Combinatorial Theory (B), 80 (2000), pp. 346-355.

[27] A. Schrijver: Combinatorial Optimization - Polyhedra and Efficiency. Springer-Verlag, 2003.

[28] Z. Svitkina and L. Fleischer: Submodular approximation: sampling-based algorithms and lower bounds. Proceedings of the 49th Annual IEEE Symposium on Foundations of Computer Science (2008), pp. 697-706.

[29] V. V. Vazirani: Approximation Algorithms. Springer-Verlag, 2001.

[30] J. Vondrák: Optimal approximation for the submodular welfare problem in the value oracle model. Proceedings of the 40th Annual ACM Symposium on Theory of Computing (2008), pp. $67-74$. 